\title{
Protocol Modeling in Spiking Neural P systems and Petri Nets
}

\author{
Venkata Padmavati \\ Metta \\ Bhilai Institute of Technology
}

\author{
Kamala Krithivasan \\ Indian Institute of Technology
}

\author{
Deepak Garg \\ Thapar University
}

\begin{abstract}
In this paper we present the relation between Spiking Neural P (SN P) systems and Petri nets by focusing on modeling simplex stop-and-wait protocol. The SN P system for the protocol is constructed and also translated it into equivalent Petri net with a corresponding semantics. It is then observed a direct correspondence between the Petri net representation of the proposed model and standard solution based on Petri nets already present in the literature.
\end{abstract}

The full text of the article is not available in the cache. Kindly refer the IJCA digital library at www.ijcaonline.org for the complete article. In case, you face problems while downloading the full-text, please send a mail to editor at editor@ijcaonline.org 